\title{
HARMONIC MEASURES ON COVERS OF COMPACT SURFACES OF NONPOSITIVE CURVATURE
}

\author{
M. BRIN AND Y. KIFER
}

\begin{abstract}
Let $M$ be the universal cover of a compact nonflat surface $N$ of nonpositive curvature. We show that on the average the Brownian motion on $M$ behaves similarly to the Brownian motion on negatively curved manifolds. We use this to prove that harmonic measures on the sphere at infinity have positive Hausdorff dimension and if the geodesic flow on $N$ is ergodic then the harmonic and geodesic measure classes at infinity are singular unless the curvature is constant.
\end{abstract}

\section{INTRODUCTION}

Let $M$ be a smooth complete, simply connected $d$-dimensional Riemannian manifold with all sectional curvatures nonpositive. There is a natural geometric compactification of $M$ by the sphere at infinity $S(\infty)$ which is the set of classes of asymptotic geodesics. Let $\Delta$ be the Laplace-Beltrami operator on $M$; then $C^{2}$-functions on $M$ satisfying $\Delta h=0$ are called harmonic. It is known that if $M$ has bounded geometry, geodesics diverge exponentially fast, and $\Delta \rho \geq$ const $>0$ with $\rho(x)=\operatorname{dist}\left(x_{0}, x\right)$, then there is a one-to-one correspondence between continuous functions $f$ on $S(\infty)$ and harmonic functions $h_{f}$ having a continuous extension to $S(\infty)$ given by the formula (see, for instance, [Ki]),

$$
h_{f}(x)=\int_{S(\infty)} f(\zeta) P(x, d \zeta)
$$

where $P(x, \cdot), x \in M, P(x, S(\infty))=1$, are the so-called harmonic measures. Let $S_{x} M$ be the sphere of unit vectors from the tangent space $T_{x} M$. Define $\Phi_{x}: S_{x} M \rightarrow S(\infty)$ which maps a vector $\zeta \in S_{x} M$ to the end at $\infty$ of the geodesic with initial velocity $\zeta$. It turns out (see [Ka2, L]) that, in general, the measure $\nu_{x}=\Phi_{x}^{-1} P(x, \cdot)$ on $S_{x} M$ is singular with respect to the Lebesgue measure on $S_{x} M$. But still under the conditions of [Ki], which hold true if all sectional curvatures of $M$ are bounded from above and below by two negative constants, the Hausdorff dimension of $\nu_{x}$ is positive (see [KL]).

Of course, these conditions fail to be true without some kind of negative curvature assumption. Nevertheless, if $M$ is the universal cover of a compact

Received by the editors August 15, 1990 and, in revised form, September 13, 1991.

1991 Mathematics Subject Classification. Primary 58G32; Secondary 30F15, 31A15.

Key words and phrases. Harmonic measures, Brownian motion, nonpositive curvature.

Both authors were partially supported by U.S.-Israel BSF.

The first author is partially supported by NSF DMS-9104134. 
rank-one manifold $N$ of nonpositive curvature with the metric on $M$ lifted from $N$, then by [B] relation (1.1) still gives a one-to-one correspondence between harmonic functions $h_{f}$ on $M$ and their continuous boundary values $f$ on $S(\infty)$, and so in this case as well one can speak of harmonic measures. The natural question whether these harmonic measures (i.e., the corresponding measures $\left.\Phi_{x}^{-1} P(x, \cdot)\right)$ have positive Hausdorff dimensions will be answered positively in this paper only in the two-dimensional case. The reason is that our argument uses global exponential divergence of geodesics which in dimension two is the same as the exponential growth of volume on geodesic spheres. The other condition $\Delta \rho>$ const $>0$ which was used in [KL] to derive the positivity of the Hausdorff dimensions of harmonic measures does not hold true everywhere in our circumstances. However, the argument actually uses only the positivity of the radial drift of the Brownian motion $X(t)$ on $M$ and this holds true in a certain average sense on covers of compact manifolds of nonpositive curvature with all sectional curvatures negative at a point. These ideas enable us to employ the machinery from [KL] and prove the positivity of the Hausdorff dimensions, as well as to modify the method of [L] in order to prove the following extension of [ $\mathrm{Ka} 2$ and $\mathrm{L}]$.

If $M$ is the universal cover of a compact nonflat surface of nonpositive curvature $N$ and the geodesic flow on $N$ is ergodic, then the measure $\nu_{x}=$ $\Phi_{x}^{-1} P(x, \cdot)$ is equivalent to the Lebesgue measure on $S_{x} M$ if and only if $N$ has constant curvature. Concerning the ergodicity assumption note that though the proof in $[\mathrm{BB}]$ contains a gap where they use an unproven assertion from $[\mathrm{P}]$, it is believed that the geodesic flow on a nonflat surface of nonpositive curvature is ergodic.

Our results admit the following purely geometric interpretation. Let $M$ be the universal cover of a nonflat surface $N$ of nonpositive curvature. Then $M$ has a standard representation as the Poincaré open unit disk $D$ with boundary $S(\infty)$ and center $\mathscr{O}$. By the uniformization theorem, $M$ is conformally equivalent to $D$ with the Euclidean metric; the Brownian motion on $M$ differs from the usual (Euclidean) Brownian motion in $D$ by a time change, and hence harmonic measures for these Brownian motions are the same. In particular, $P(\mathscr{O}, \cdot)$ is the normalized Lebesgue measure on $S(\infty)$. For each $\gamma \in S(\infty)$, let $\xi=\xi_{\gamma} \in S_{\mathscr{O}} D$ be the direction of the geodesic in the metric inherited from $M$ connecting $\gamma$ and $\mathscr{O}$ and let $\eta(\xi) \in S_{\mathscr{O}} D$ be the direction of the Euclidean radius connecting $\gamma$ and $\mathscr{O}$. Then $\eta: S_{\mathscr{O}} D \rightarrow S_{\mathscr{O}} D$ is a homeomorphism of the unit circle. The singularity of harmonic measures means that $\eta$ maps the Lebesgue measure to a singular measure (assuming that the geodesic flow on $N$ is ergodic) unless the curvature of $M$ is constant; however, the Hausdorff dimension of this measure is positive.

By the Harnack inequality it is easy to see (see, e.g., [Ki]) that all harmonic measures are equivalent, and so one has a harmonic measure class. The map $\Phi_{x}$ projects the normalized Lebesgue measure $m_{x}$ on $S_{x} M$ to a probability measure $\mu_{x}$ on $S(\infty)$. If $M$ is the universal cover of a compact manifold $N$ of negative curvature, then it is well known that measures $\mu_{x}$ for all $x \in M$ are equivalent, and so one has a geodesic measure class. In the nonpositive curvature case whether or not the measures $\mu_{x}$ are equivalent remains an open question since $[\mathrm{BB}]$ contains a gap in the proof though it is believed to be true, 
at least in the two-dimensional case. This is equivalent to the ergodicity of the geodesic flow on $N$. We will prove here under this condition that all harmonic measures $P(x, \cdot), x \in M$, are singular with respect to all $\mu_{y}, y \in M$, unless the curvature is constant.

\section{GeOMETRIC PRELIMINARIES}

We assume that $M$ is the universal cover of a compact manifold $N$ of nonpositive sectional curvature. By the Cartan-Hadamard theorem the exponential map $\operatorname{Exp}_{x}: T_{x} M \rightarrow M$ based at any point $x \in M$ is a diffeomorphism of $T_{x} M$ onto $M$. Hence there is a global system of geodesic polar coordinates which assigns to each $z \in M$ the pair $(r, \xi)$ where $r=\operatorname{dist}(x, z), \xi \in S_{x} M, z=$ $\operatorname{Exp}_{x}(r \xi)$, and dist denotes the Riemannian distance. In these coordinates the Laplace-Beltrami operator can be written in the form

$$
\Delta=\frac{\partial^{2}}{\partial r^{2}}+Q_{x}(r, \xi) \frac{\partial}{\partial r}+\Delta_{\xi}
$$

where $\Delta_{\xi}$ does not contain derivatives in $r$, and

$$
Q_{x}(r, \xi)=\frac{\partial}{\partial r}\left(\log A_{x}(r, \xi)\right)=\Delta \rho_{x}(r)>0 .
$$

Here $\rho_{x}$ is the distance function from $x$ and $A_{x}(r, \xi)$ is the $(d-1)$-dimensional volume element on the geodesic sphere $S_{x}(r)$ of radius $r$ centered at $x$ (see [IW, $\S \mathrm{VI} .5]$ and [Ch, p. 67]). Note that $Q(r, \xi)$ is the trace of the operator of the second fundamental form of the sphere $S_{x}(r)$ (see [G, Chapter 3]). Since $M$ is the universal cover of a compact manifold $N$, the Ricci curvature of $M$ is bounded below. Hence, by Bishop's comparison theorem (see [Ch, pp. 7173]), $Q_{x}(r, \xi)$ is uniformly bounded from above for all $r \geq \delta>0$. Let $B_{x}(r)$ denote the ball of radius $r$ centered at $x$ in $M$ or in $N$.

2.1. Lemma. Let $M$ be the universal cover of a compact manifold $N$ of nonpositive curvature of rank 1 (see [BB]). Then there exist $R_{0}, b_{0}, r_{0}>0$ such that for any $x, y \in M$ there is $z_{y} \in B_{y}\left(R_{0}\right)$ with the property $Q_{x}(r, \xi) \geq b_{0}$ for any $(r, \xi) \in B_{z_{y}}\left(r_{0}\right)$.

Proof. Let us show first that there are $R_{0}, \tilde{b}_{0}>0$ such that for any $x, y \in M$ there exists $z_{y} \in B_{y}\left(R_{0}\right)$ for which $Q_{x}(r, \xi) \geq \tilde{b}_{0}$ with $(r, \xi)=z_{y}$. Suppose not. Then there are sequences $x_{k}, y_{k} \in M$ such that

$$
Q_{x_{k}}(r, \xi)<\frac{1}{k}
$$

for any $(r, \xi) \in B_{y_{k}}(k)$. Obviously $d_{k}=\operatorname{dist}\left(x_{k}, y_{k}\right) \rightarrow \infty$ as $k \rightarrow \infty$ because $Q_{x}(r, \xi) \geq(d-1) / r$, where $d=\operatorname{dim} M$. Since $M$ is the universal cover of a compact manifold, there is a sequence of isometries $\theta_{k}$ for which the points $\theta_{k} y_{k}$ lie in the same fundamental domain. Set $x_{k}^{\prime}=\theta_{k} x_{k}, y_{k}^{\prime}=\theta_{k} y_{k}$ and choose a subsequence $n_{k} \rightarrow \infty$ such that

$$
\text { (i) } x_{n_{k}}^{\prime} \rightarrow \bar{x} \in S(\infty), \quad \text { (ii) } y_{n_{k}} \rightarrow \bar{y} \in M \text {. }
$$

The horosphere $H_{\bar{x}}(\bar{y})$ passing through $\bar{y}$ and centered at $\bar{x}$ is uniformly approximated in the $C^{2}$-topology (on compact subsets) by the spheres $S_{x_{n_{k}}}\left(d_{n_{k}}\right)$, see [HI]. Let $V_{\bar{x}}(z)$ be the operator of the second fundamental form of $H_{\bar{x}}(\bar{y})$ 
at $z \in H_{\bar{x}}(\bar{y})$. Since the curvature of $M$ is nonpositive, $V_{\bar{x}}(z)$ is a nonnegative selfadjoint operator. By (2.3) and (2.4), $\operatorname{tr} V_{\bar{x}}(z)=0$ for any $z \in H_{\bar{x}}(\bar{y})$. Hence,

$$
V_{\bar{x}}(z)=0 \text { for any } z \in H_{\bar{x}}(\bar{y}) .
$$

It follows now from [CE] that $M$ admits a Riemannian splitting $M=M^{\prime} \times \mathbb{R}^{1}$ and has rank at least 2 , which contradicts the assumption and proves the claim above.

Since the spheres approximate the horospheres in the $C^{2}$-topology uniformly on compact subsets [HI], the functions $Q_{x}(r, \xi)$ are uniformly equicontinuous for $r>\delta$ for any $\delta>0$. Therefore, we can choose $b_{0}=\tilde{b}_{0} / 2$ and the lemma follows.

2.1.a. Remark. Suppose that for some point $x_{0} \in N$ all sectional curvatures are negative; this happens for example if $N$ is a rank 1 surface. There are $\tilde{r}>0$ and $a^{2}>0$ such that for any $x \in B_{x_{0}}(r)$ the curvature of any 2-plane at $x$ is less than $-a^{2}$. Let $\pi: M \rightarrow N$ be the projection. Then in the previous lemma we can set $R_{0}$ to be the diameter of a fundamental domain, the points $z_{y}$ to be the preimages of $x_{0}$ under $\pi, r_{0}=\tilde{r} / 2, b_{0}=a(d-1) \tanh a r_{0}$, where $d=\operatorname{dim} N$.

The following lemma gives an exponential estimate on the divergence of geodesics in $M$ when $\operatorname{dim} M=2$. Denote by $\operatorname{dist}_{x}^{(r)}$ distance on the geodesic circle $S_{x}(r)$.

2.2. Lemma. Let $\operatorname{dim} M=2$. Then there exists $\gamma_{0}, 0<\gamma_{0}<1$, such that for any $r_{2} \geq r_{1} \geq 1$,

$$
\gamma_{0} e^{\gamma_{0}\left(r_{2}-r_{1}\right)} \leq \frac{\operatorname{dist}_{x}^{\left(r_{2}\right)}\left(\operatorname{Exp}_{x} r_{2} \xi, \operatorname{Exp}_{x} r_{2} \eta\right)}{\operatorname{dist}_{x}^{\left(r_{1}\right)}\left(\operatorname{Exp}_{x} r_{1} \xi, \operatorname{Exp}_{x} r_{1} \eta\right)} \leq \gamma_{0}^{-1} e^{\gamma_{0}^{-1}\left(r_{2}-r_{1}\right)}
$$

for all $x \in M, \xi, \eta \in S_{x} M$, provided

$$
\operatorname{dist}_{x}^{\left(r_{1}\right)}\left(\operatorname{Exp}_{x} r_{1} \xi, \operatorname{Exp}_{x} r_{1} \eta\right) \geq 2 R_{0}
$$

(See Lemma 2.1 for the definition of $R_{0}$.)

Proof. Put

$$
C_{x}(r, \xi)=\left\{\eta \in S_{x} M: \operatorname{dist}_{x}^{(r)}\left(\operatorname{Exp}_{x} r \xi, \operatorname{Exp}_{x} r \eta\right)<R_{0}\right\}
$$

and

$$
S_{x}(\xi, \rho)=\left\{\operatorname{Exp}_{x}(\rho+s) \eta: \eta \in C_{x}(\rho, \xi), 0 \leq s \leq 2 R_{0}\right\} .
$$

Note that if the length of the geodesic circle $S_{x}(\rho)$ is less than $2 R_{0}$, then the truncated sector $S_{x}(\xi, \rho)$ is an annulus. Put $y=\operatorname{Exp}_{x}\left(\rho+R_{0}\right) \xi$. Clearly $B_{y}\left(R_{0}\right) \subset B_{x}\left(\rho+2 R_{0}\right) \backslash B_{x}(\rho)$. Note that if a curve lies outside the circle $S_{x}(\rho)$, then its radial projection onto $S_{x}(\rho)$ is shorter than the curve itself. It follows that

$$
S_{x}(\xi, \rho) \supset B_{y}\left(R_{0}\right)
$$

Set for brevity

$$
\operatorname{dist}_{x}^{\left(r_{i}\right)}\left(\operatorname{Exp}_{x} r_{i} \xi, \operatorname{Exp}_{x} r_{i} \eta\right)=\operatorname{dist}_{i}, \quad i=1,2 .
$$


Let $m=\left[\operatorname{dist}_{1} / 2 R_{0}\right] \geq 1$ and $N=\left[\left(r_{2}-r_{1}\right) / 2 R_{0}\right]$, where [ ] denotes the integer part. Consider the arc of $S_{x}\left(r_{1}\right)$ with ends $\operatorname{Exp}_{x} r_{1} \xi$ and $\operatorname{Exp}_{x} r_{1} \eta$. Divide it into subarcs $a_{i}=a_{i}\left(r_{1}\right), i=1,2, \ldots, m$, of length $2 R_{0}$ and the remainder $a^{\prime}=a^{\prime}\left(r_{1}\right)$ whose length $\Delta$ is less than $2 R_{0}$. Let $a_{i}(r)$ denote the radial projection of $a_{i}$ onto $S_{x}(r)$. Since the curvature is nonpositive, the lengths of each $a_{i}(r)$ and of $a^{\prime}(r)$ are strictly increasing functions of $r$. By (2.6), each subarc $a_{i}$ encounters at least $n$ times balls $B_{z_{y}}\left(r_{0}\right)$ (see Lemma 2.1) as it moves radially from $S_{x}\left(r_{1}\right)$ to $S_{x}\left(r_{2}\right)$. For each such encounter there are $\zeta \in T_{x} M$ and $r, r_{1}<r<r_{2}$, such that $\operatorname{Exp}_{x} r \zeta$ lies in $a_{i}(r)$ and is one of the points $z_{y}$ from Lemma 2.1. Therefore the logarithmic derivative $Q_{x}\left(r^{\prime}, \zeta^{\prime}\right)$ of the length element $A_{x}\left(r^{\prime}, \zeta^{\prime}\right)$ (see (2.2)) is at least $b_{0}$ whenever $\operatorname{dist}\left(\operatorname{Exp}_{x} r^{\prime} \zeta^{\prime}, \operatorname{Exp}_{x} r \zeta\right) \leq r_{0}$. Thus length $\left(a_{i}\left(r_{2}\right)\right) \geq \exp (\delta n) \cdot \operatorname{length}\left(a_{i}\left(r_{1}\right)\right)$, where $\delta>0$ does not depend on $x, r_{1}, r_{2}, \xi, \eta$. For $r_{2}-r_{1} \geq 2 R_{0}$ we have

$$
\frac{\text { dist }_{2}}{\text { dist }_{1}} \geq \frac{2 R_{0} m \exp (\delta n)+\Delta}{2 R_{0} m+\Delta}>e^{\delta n / 2} \geq \exp \left(\delta\left(r_{2}-r_{1}\right) / 4 R_{0}\right) .
$$

Since dist dist $_{1}$, the lower bound of the lemma follows for $r_{2}-r_{1}<2 R_{0}$ as well, with a properly chosen $\gamma_{0}$. The upper bound holds true since the curvature of $M$ is bounded from below (see Lemma 4.2 in [BBE]).

2.3. Remark. For dimensions greater than 2, Lemma 2.1, Formula (2.2) and arguments similar to those in the proof of Lemma 2.2 imply the exponential growth of volume on spheres $S_{x}(r)$ instead of length. In this case, exponential divergence of geodesics, which we use later, fails in general.

Recall that the geodesic flow in the unit tangent bundle $S N$ is the oneparameter group of transformations $g^{t}$ which parallel translates every unit vector $\xi$ distance $t$ along the geodesic with initial velocity $\xi$. The flow $g^{t}$ preserves the Liouville measure $d m_{x}(\xi) d m(x)$, where $m$ is the Riemannian volume in $M$ and $N$ and $m_{x}$ is the Lebesgue measure on the spheres $S_{x} N$ and $S_{x} M$

2.4. Lemma, Let $\operatorname{dim} M=2$ and assume that the geodesic flow $g^{t}$ is ergodic. For $\xi \in S_{x} M$ let $A_{\delta}(t, x, \xi)=\left\{\eta \in S_{x} M: \operatorname{dist}\left(\operatorname{Exp}_{x} t \xi, \operatorname{Exp}_{x} t \eta\right) \leq \delta\right\}$. Then for any $\delta>0$ and almost all $(x, \xi)$ with respect to the Liouville measure

$$
\lim _{t \rightarrow \infty} \frac{1}{t} \log m_{x}\left(A_{\delta}(t, x, \xi)\right)=-\gamma_{1},
$$

where $\gamma_{1}>0$ is the positive Lyapunov exponent of the geodesic flow.

Proof. Let $\xi, \eta \in S_{x} M$. Set $d(t)=\operatorname{dist}\left(\operatorname{Exp}_{x} t \xi, \operatorname{Exp}_{x} t \eta\right)$. By the second variation formula $d^{\prime \prime}(t) \leq-\min K \cdot d(t)$. In particular, $d(t)$ is convex, so that $d(t) \geq t \cdot d^{\prime}(0), t \geq 0$. We have

$$
d^{\prime}(t) d^{\prime \prime}(t) \leq-\min K \cdot d^{\prime}(t) d(t),
$$

and hence

$$
\left(d^{\prime}(t)\right)^{2} \leq\left(d^{\prime}(0)\right)^{2}+(d(t))^{2}(-\min K) .
$$

Let $\gamma_{t}$ be the geodesic connecting $x_{1}=\operatorname{Exp}_{x} t \xi$ and $x_{2}=\operatorname{Exp}_{x} t \eta$ and let $v_{i} \in S_{x_{i}} M, i=1,2$, be unit vectors perpendicular to $\gamma_{t}$ and pointing away from $x$. Let $\alpha<\frac{\pi}{2}$ be the angle between $g^{t} \xi$ and $v_{1}$ and $\beta<\frac{\pi}{2}$ be the angle 
between $g^{t} \eta$ and $v_{2}$. Then $d^{\prime}(t)=\sin \alpha+\sin \beta$ and hence, using $\sin s \geq \frac{2}{\pi} s$ for $0 \leq s \leq \frac{\pi}{2}$,

$$
\alpha+\beta \leq \frac{\pi}{2} d^{\prime}(t) .
$$

Thus, for $t \geq 1$,

$$
\begin{aligned}
\operatorname{dist}_{S M}\left(g_{\xi}^{t}, g_{\eta}^{t}\right) & \leq(\alpha+\beta)^{2}+(d(t))^{2} \\
& \leq \frac{\pi^{4}}{4}\left[\left(d^{\prime}(0)\right)^{2}+(d(t))^{2} \cdot(-\min K)\right]+(d(t))^{2} \\
& \leq 3(2-\min K) \cdot(d(t))^{2} .
\end{aligned}
$$

Let $y(t, \eta)$ be the length of the perpendicular Jacobi field along the geodesic $\operatorname{Exp}_{x} t \eta$ with initial conditions $y(0, \eta)=0, y^{\prime}(0, \eta)=1$. Let $u(t, \eta)=$ $y^{\prime}(t, \eta) / y(t, \eta)$ be the geodesic curvature of the geodesic circle $S_{x}(t)$ at $\operatorname{Exp}_{x} t \eta$. Since the curvature is nonpositive, $u(t, \eta)>0$. Let $U\left(g^{t} \eta\right)$ denote the geodesic curvature of the unstable horocycle of $g^{t} \eta$. By [HI], the geodesic circles converge uniformly on compact sets in the $C^{2}$-topology to the unstable horocycles, and $u(t, \eta)-U\left(g^{t} \eta\right) \searrow 0$ as $t \rightarrow \infty$. Fix $\varepsilon>0$ and let $T_{0}>1$ be such that $\left|u(t, \eta)-U\left(g^{t} \eta\right)\right|<\varepsilon$ for any $t \geq T_{0}$, and $x \in M$ and $\eta \in S_{x} M$.

Since the function $U(\zeta), \zeta \in S M$, is uniformly continuous, there is $\delta_{1}>0$ such that $\left|U\left(\zeta_{1}\right)-U\left(\zeta_{2}\right)\right|<\varepsilon$ provided $\operatorname{dist}_{S M}\left(\zeta_{1}, \zeta_{2}\right)<\delta_{1} \sqrt{3(2-\min K)}$. Therefore, by (2.7)

$$
|u(t, \eta)-u(t, \xi)|<3 \varepsilon
$$

for $\xi, \eta \in S_{x} M, t \geq T_{0}, \operatorname{dist}\left(\operatorname{Exp}_{x} t \xi, \operatorname{Exp}_{x} t \eta\right)<\delta_{1}$. Hence

$$
\left|\ln \left(\frac{y(t, \eta)}{y\left(T_{0}, \eta\right)} / \frac{y(t, \xi)}{y\left(T_{0}, \xi\right)}\right)\right|<3 \varepsilon\left(t-T_{0}\right) .
$$

By the multiplicative ergodic theorem a.e. $\xi \in S M$ satisfies

$$
\lim _{t \rightarrow \infty} \frac{1}{t} \log y(t, \xi)=\gamma_{1} .
$$

Choose $\xi$ to be such a vector and assume also that $\xi$ is uniformly recurrent under the geodesic flow, i.e., for any set $F$ of positive measure in $S N$,

$$
\lim _{t \rightarrow \infty} \frac{1}{t} \int_{0}^{t} \chi_{F}\left(g^{\tau} \xi\right) d \tau=\mu(F),
$$

where $\chi$ denotes the characteristic function and $\mu$ the Liouville measure. Since $N$ is not flat, there is a point $y \in N$ where the curvature is negative. Let $F$ be the set of tangent vectors whose foot points have the property that the curvature of $N$ is less than $-\delta_{2}$ in the $\delta_{2}$-neighborhood of the point. Then each time the geodesic $\operatorname{Exp}_{x} t \xi$ passes those points, a finite arc of the geodesic circle $S_{x}(t)$ (of length $\delta_{2}$ ) is stretched at least by a constant factor greater than 1. Let $\eta \in A_{\delta}(t, x, \xi)$. Then, by (2.10), there is $T_{1}>0$ such that

$$
\operatorname{dist}_{x}^{(\tau)}\left(\operatorname{Exp}_{x} \tau \xi, \operatorname{Exp}_{x} \tau \eta\right)<\delta_{1}
$$

for $\tau=t(1-\varepsilon), t \geq t_{1}$. By combining (2.8), (2.9) and (2.11) we get

$$
\left|\log y(t, \eta)-\gamma_{1} t\right| \leq C_{1}+C_{2} \varepsilon t
$$


for some constants $C_{1}, C_{2}$ and any $\eta \in A_{\delta}(t, \xi)$ provided $t$ is large enough. Note that

$$
\operatorname{dist}_{x}^{(t)}\left(\operatorname{Exp}_{x} t \xi, \operatorname{Exp}_{x} t \eta\right)=\int_{\xi}^{\eta} y(t, \zeta) d m_{x}(\zeta)
$$

Since $N$ is compact, the absolute value of the curvature is bounded, and hence the geodesic curvature of all geodesic circles with radius at least 1 is bounded from above. Therefore there is $C_{3}>0$ such that

$$
\operatorname{dist}_{x}^{(t)}\left(\operatorname{Exp}_{x} t \xi, \operatorname{Exp}_{x} t \eta\right) \leq C_{3} \operatorname{dist}\left(\operatorname{Exp}_{x} t \xi, \operatorname{Exp}_{x} t \eta\right)
$$

if the distance in the right-hand side is at most $\delta_{1}$ and $t \geq 1$. By (2.13) and (2.14), for any $\eta \in A_{\delta}(t, x, \xi)$ we have

$$
\begin{aligned}
C_{3}^{-1} e^{\gamma_{1} t-C_{1}-C_{2} \varepsilon t} \int_{\xi}^{\eta} d m_{x}(\xi) & \leq C_{3}^{-1} \operatorname{dist}_{x}^{(t)}\left(\operatorname{Exp}_{x} t \xi, \operatorname{Exp}_{x} t \eta\right) \\
& \leq \operatorname{dist}\left(\operatorname{Exp}_{x} t \xi, \operatorname{Exp}_{x} t \eta\right) \\
& \leq \operatorname{dist}^{(t)}\left(\operatorname{Exp}_{x} t \xi, \operatorname{Exp}_{x} t \eta\right) \\
& \leq e^{\gamma_{1} t+C_{1}+C_{2} \varepsilon t} \int_{\xi}^{\eta} d m_{x}(\xi)
\end{aligned}
$$

which proves the lemma.

2.5. Remark. The geodesic flow on a compact surface of nonpositive curvature is ergodic if the set of those geodesics along which the curvature is identically zero has Liouville measure zero. Whether this holds true for any nonflat compact surface is an open question. We describe now an example of a surface of nonpositive but not negative curvature for which the geodesic flow is ergodic. Consider two flat tori $T_{1}, T_{2}$ which we view as unit squares with opposite sides identified. Remove two discs and connect their boundary circles by a "neck" of negative curvature (see Figure 1). The curvature of the resulting surface (of genus 2 ) is 0 on the tori and negative on the connecting neck. Obviously every geodesic that starts on one of the tori with an irrational slope will eventually enter the neck. Also every geodesic that starts in the neck will eventually leave the neck. Therefore along a.e. geodesic the curvature is not identically 0 .
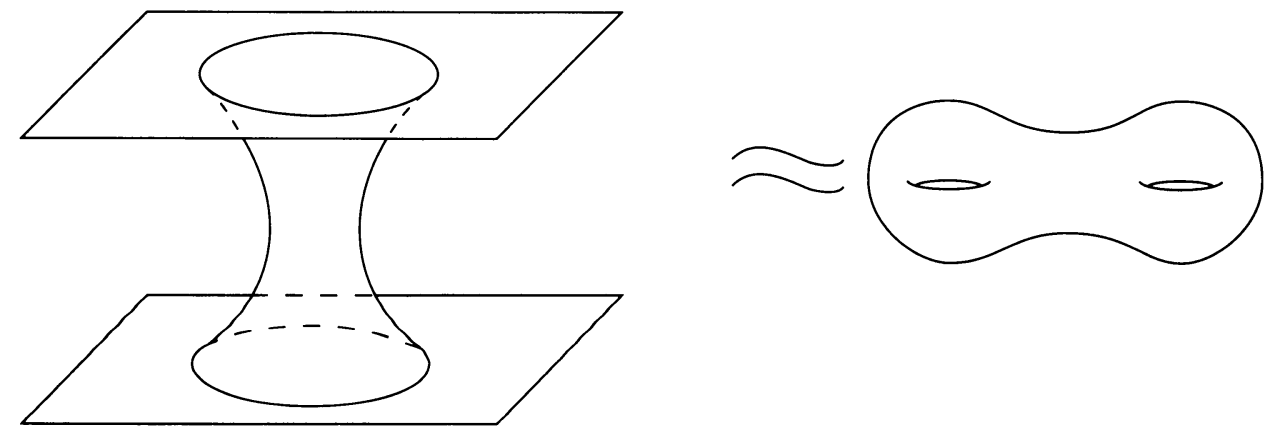

FIGURE 1 
2.6. Remark. The absolute continuity of the weak stable horospheric foliation for $M$, by definition, implies that all the measures $\mu_{x}$ from $\S 1$ are equivalent. The ergodicity of the geodesic flow $g$ on a rank 1 compact manifold $N$ is equivalent to the absolute continuity of the weak stable horospheric foliation on $M$. Namely, if $g$ is ergodic then almost every orbit of $g$ visits the open subset of $S N$ corresponding to geodesics with no parallel Jacobi fields. Hence the Lyapunov exponents of $g$ are nonzero almost everywhere and the weak stable horospheric foliation is absolutely continuous [P]. Conversely, if the horospheric foliation is absolutely continuous, then the geodesic flow is ergodic by [P].

2.7. Remark. P. Eberlein informed us that the estimate of Lemma 2.2 holds true for all simply connected uniform visibility manifolds with a compact quotient in any dimension. This exponential divergence of geodesics is essentially equivalent to the manifold being a negatively curved metric space in the sense of M. Gromov. Together with Lemma 2.1 it implies that the method of the present paper works for all such manifolds. Hence the Martin boundary for such manifolds coincides with the sphere at infinity and the Hausdorff dimension of harmonic measures is positive. Note that all nonflat simply connected nonpositively curved surfaces with a compact quotient are always uniform visibility manifolds and negatively curved metric spaces in the sense of Gromov.

\section{Probabilistic lemmas}

Recall that the Brownian motion $X(t)$ on $M$ is a diffusion process generated by the Laplace-Beltrami operator $\Delta$ (see [IW, Chapters V and VI]). In the geodesic polar coordinates with a pole at $x$ one can write $X(t)=$ $\left(R_{x}(t), \Xi_{x}(t)\right)$, where $\Xi_{x}(t) \in S_{x} M$ and $X(t)=\operatorname{Exp}_{x}\left(R_{x}(t) \Xi_{x}(t)\right)$. Let $\tau_{x}(r)=$ $\inf \left\{t \geq 0: X(t) \in S_{x}(r)\right\}$, i.e., $\tau_{x}(r)$ is the first hitting time by $X(t)$ of the geodesic sphere $S_{x}(r)$. Let $a \wedge b=\min (a, b)$ and $E_{y}, P_{y}$ denote the expectation and probability for the process $X(t)$ starting at $y$.

3.1. Lemma. For all $y \in M, t \geq 0$ and $\delta>0$,

$$
E_{y} R_{x}(t) \geq \operatorname{dist}(x, y)+b_{0} \delta P_{y}\left\{\tau_{2} \wedge t \geq \tau_{1}+\delta\right\},
$$

where $\tau_{1}=\tau_{z_{y}}\left(r_{0} / 2\right), \tau_{2}=\inf \left\{s>\tau_{1}: X(s) \in S_{z_{y}}\left(r_{0}\right)\right\}, z_{y}, r_{0}, b_{0}$ are the same as in Lemma 2.1.

Proof. Since the Laplace-Beltrami operator $\Delta$ has the form (2.1) in geodesic polar coordinates, the radial part $R_{x}(t)$ of $X(t)$ satisfies the following stochastic equation

$$
R_{x}(t)=R_{x}(0)+\sqrt{2} w(t)+\int_{0}^{t} Q_{x}(X(s)) d s,
$$

where $w(t)$ is the one-dimensional Wiener process starting at zero. Furthermore,

$$
\begin{aligned}
R_{x}(t)-R_{x}(0)= & \sqrt{2} w(t)+\int_{0}^{\tau_{1} \wedge t} Q_{x}(X(s)) d s \\
& +\chi_{\tau_{1}<t} \int_{\tau_{1}}^{\tau_{2} \wedge t} Q_{x}(X(s)) d s+\int_{\tau_{2} \wedge t}^{t} Q_{x}(X(s)) d s \\
\geq & \sqrt{2} w(t)+b_{0}\left(\tau_{2} \wedge t-\tau_{1}\right) \chi_{\tau_{1}<t},
\end{aligned}
$$


where $\chi_{A}$ denotes the indicator of event $A$. Taking the expectation of both parts in (3.3), one gets (3.1).

3.2. Lemma. There exists $\alpha>0$ such that for any $x \in M$ with probability one

$$
\lim _{t \rightarrow \infty} \frac{1}{t} \operatorname{dist}(x, X(t))=\lim _{t \rightarrow \infty} \frac{1}{t} R_{x}(t)=\alpha .
$$

Proof. The existence of the limit in (3.4) is known (see [Va, $\S I I .7])$ whenever $M$ is the universal cover of a compact manifold $N$. The argument goes as follows. Let $\pi: M \rightarrow N$ be the natural projection. Then $\pi X_{t}$ is the Brownian motion on $N$. It preserves the normalized volume on $N$ which is the unique probability invariant measure of $\pi X_{t}$, and so it can be considered as an ergodic stationary process. Let $\theta_{s}: \Omega \rightarrow \Omega$ be the corresponding time shift so that $\pi X\left(t, \theta_{s} \omega\right)=\pi X(t+s, \omega)$. Any continuous path $\varphi_{s}, 0 \leq s \leq t$, on $N$ can be uniquely lifted to $M$ provided we fix a fundamental domain $D$ on $M$ where $\varphi_{0}$ should be mapped. Hence for each $s>0$ we can define uniquely $X\left(t, \theta_{s} \omega\right)$ with $X\left(0, \theta_{s} \omega\right) \in D$ lifting the path $\pi X\left(u, \theta_{s} \omega\right), 0 \leq u \leq t$ from $N$ to $M$. Thus if $X(0, \omega) \in D$ is a random point whose distribution is the normalized Riemannian volume on $D$, then $g(t, \omega)=\operatorname{dist}(x(0, \omega), X(t, \omega))$ is a stationary ergodic process. By the triangle inequality $g(t+s, \omega) \leq g(t, \omega)+g\left(s, \theta_{t} \omega\right)$ since $X\left(0, \theta_{t} \omega\right)=X(t, \omega)$ and $X\left(s, \theta_{t} \omega\right)=X(t+s, \omega)$. By Kingman's subadditive ergodic theorem the limit in (3.4) exists for almost all $x=X(0, \omega)$ in $D$ with probability one. Let $x$ be any point in $D$ and $X(0, \omega) \equiv x$. Then for each $s>0$,

$$
\begin{aligned}
\lim _{t \rightarrow \infty} & \frac{1}{t} \operatorname{dist}(x, X(t, \omega))=\lim _{t \rightarrow \infty} \frac{1}{t} \operatorname{dist}(x(s, \omega), X(t, \omega)) \\
= & \lim _{t \rightarrow \infty} \frac{1}{t} \operatorname{dist}\left(x\left(0, \theta_{s} \omega\right), X\left(t-s, \theta_{s} \omega\right)\right) \stackrel{\text { def }}{=} \alpha
\end{aligned}
$$

with probability one since the distribution of $X\left(0, \theta_{s} \omega\right)$ on $D$ is equivalent to the volume. Thus the limit (3.4) exists, is the same for all $x \in D$, and, by its invariance under isometries, is the same for all $x \in M$.

To show that $\alpha>0$ it suffices to derive that

$$
\lim _{t \rightarrow \infty} \frac{1}{t} E_{x} R_{x}(t)=\alpha>0 .
$$

The limit in (3.6) exists by the subadditivity. By Lemma 3.1,

$$
E_{y} R_{x}(1) \geq \operatorname{dist}(x, y)+b_{1}
$$

for some $b_{1}>0$ independent of $x$ and $y$. Thus by the Markov property of the Brownian motion

$$
E_{x} R_{x}(n)=E_{x} E_{X(n-1)} R_{x}(1) \geq E_{x} R_{x}(n-1)+b_{1} .
$$

Repeating this argument we obtain $E_{x} R_{x}(n) \geq n b_{1}$ which implies that $\alpha>$ 0 .

3.3. Lemma. There exists $\kappa \in(0,1)$ such that for any $x, y \in M$ and $t \geq 1$,

$$
P_{y}\left\{\kappa s \leq R_{x}(s)-R_{x}(0) \leq \kappa^{-1} s \text { for all } s \geq t\right\} \geq 1-\kappa^{-1} e^{-\kappa t} .
$$

Proof. Let $z_{y}, r_{0}$ and $b_{0}$ be the same as in Lemma 2.1. We may assume that $t$ is big enough. Introduce the following stopping times $\tau_{0}=0, \tau_{2 j+1}=\inf \{t \geq$ 
$\left.\left.j: \operatorname{dist}(X(t)), z_{X(j)}\right) \leq r_{0} / 2\right\}, \quad \tau_{2 j+2}=\inf \left\{t \geq \tau_{2 j+1}: \operatorname{dist}\left(X(t), z_{X(j)}\right) \geq r_{0}\right\}$ for $j=0,1, \ldots$. Denote by $A_{j+1}$ the event $\left\{j \leq \tau_{2 j+1} \leq j+\frac{1}{3}\right.$ and $j+\frac{2}{3} \leq$ $\left.\tau_{2 j+1}<j+1\right\}$ for $j=0,1, \ldots$. Put

$$
\mathscr{A}_{l}^{k}=\bigcup_{(l-1) k<j \leq l k} A_{j} .
$$

Then by (3.2),

$$
\begin{aligned}
R_{x}(t) & \geq R_{x}(0)+\sqrt{2} w(t)+\sum_{1 \leq j \leq t} \int_{j-1}^{j} Q_{x}(X(s)) d s \\
& \geq R_{x}(0)+\sqrt{2} w(t)+\frac{1}{3} b_{0} \sum_{1 \leq j \leq t} \chi_{A_{j}} \\
& \geq R_{x}(0)+\sqrt{2} w(t)+\frac{1}{3} b_{0} \sum_{1 \leq l \leq l t / k]} \chi_{\mathscr{A}_{l}^{k}},
\end{aligned}
$$

where $[a]$ denotes the integral part of $a$. For any $0<l_{1}<l_{2}<\cdots<l_{n} \leq$ $[t / k]$, let $\mathscr{E}_{l_{1}, \ldots, l n}^{k}$ be the event that all $\mathscr{A}_{l_{1}}^{k}, \mathscr{A}_{l_{2}}^{k}, \ldots, \mathscr{A}_{l_{n}}^{k}$ occur and if $l \neq l_{i}$, $0<l \leq[t / k]$ for each $i=1, \ldots, n$, then the negation of $\mathscr{A}_{l}{ }^{k}$ occurs, i.e.,

$$
\mathscr{E}_{l_{1}, \ldots, l_{n}}^{k}=\left(\bigcap_{1 \leq i \leq n} \mathscr{A}_{l_{i}}^{k}\right) \cap\left(\bigcap_{l \neq l_{i}}\left(\Omega \backslash \mathscr{A}_{l_{1}}^{k}\right)\right) .
$$

By the Markov property of the Brownian motion $X(t)$,

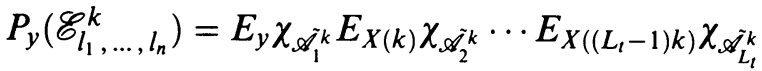

$$
\begin{aligned}
& \leq\left(1-\inf _{z \in M} P_{z}\left(\mathscr{A}_{1}^{k}\right)\right)^{L_{t}-n},
\end{aligned}
$$

where $L_{t}=[t / k]$ and $\tilde{\mathscr{A}}_{l}^{k}=\mathscr{A}_{l}^{k}$ if $l=l_{i}$ for some $i=1, \ldots, n$ and $\tilde{\mathscr{A}}_{l}^{k}=\Omega \backslash \mathscr{A}_{l}{ }^{k}$ if $l \neq l_{i}$ for all $i=1, \ldots, n$. Again by the Markov property

$$
\begin{aligned}
P_{z}\left(\mathscr{A}_{1}^{k}\right) & =1-P_{z}\left(\bigcap_{0<j \leq k}\left(\Omega \backslash A_{j}\right)\right) \\
& =1-E_{z} \chi_{\Omega \backslash A_{1}} E_{X(1)} \chi_{\Omega \backslash A_{1}} \cdots E_{X(k-1)} \chi_{\Omega \backslash A_{1}} \\
& \geq 1-\left(1-\inf _{v \in M} P_{v}\left(A_{1}\right)\right)^{k} .
\end{aligned}
$$

Since $\operatorname{dist}\left(z_{a}, a\right) \leq R_{0}$ for any $a \in M$, it follows from the strong Markov property that

$$
\begin{aligned}
P_{v}\left(A_{1}\right) \geq & \left(\inf _{w \in M} \inf _{u \in B_{w}\left(R_{0}\right)} P_{w}\left\{\tau_{u}\left(\frac{r_{0}}{2}\right)<\frac{1}{3}\right\}\right) \\
& \times\left(\inf _{w \in M} \inf _{u \in B_{w}\left(r_{0} / 2\right)}\left(P_{u}\left\{\tau_{w}\left(r_{0}\right)<1\right\}-P_{u}\left\{\tau_{w}\left(r_{0}\right)<\frac{2}{3}\right\}\right)\right) .
\end{aligned}
$$

Since $g(s, w)=P_{w}\left\{\tau_{u}(r)<s\right\}$ solves the parabolic equation $\partial g / \partial s=\Delta g$ in the geodesic ball $B_{u}(r)$ of radius $r$ centered at $u$ with initial condition 
$\left.g\right|_{s=0}=0$ and boundary condition $\left.g\right|_{S_{u}(r)}=1$, it follows that the right-hand side in (3.13) is positive. Hence, if $k \geq k_{0}$ is big enough, then the right-hand side of (3.12) is bigger than $\frac{7}{8}$, and so by (3.11),

$$
P_{y}\left(\mathscr{E}_{l_{1}, \ldots, l_{n}}^{k_{0}}\right) \leq\left(\frac{1}{8}\right)^{L_{t}-n} .
$$

Let $\mathscr{T}_{t}=\sum_{1 \leq l \leq L_{t}} \chi_{\mathscr{Q}_{1}^{k_{0}}}$. Then by (3.14),

$$
\begin{aligned}
P_{y}\left\{\mathscr{T}_{t} \leq T\right\} & \leq \sum_{0 \leq n \leq T} \sum_{l_{1}, \ldots, l_{n}} P_{y}\left(\mathscr{E}_{l_{1}, \ldots, l_{n}}^{k_{0}}\right) \\
& \leq \sum_{0 \leq n \leq T}\left(\begin{array}{c}
L_{t} \\
n
\end{array}\right)\left(\frac{1}{8}\right)^{L_{t}-n} \leq 2^{3 T-2 L_{t}} .
\end{aligned}
$$

Thus by (3.10) and the reflection principle (see [KS, p. 80]) it follows that

$$
\begin{aligned}
P_{y}\{ & \left.R_{x}(s)-R_{x}(0) \leq \frac{b_{0} s}{36 k_{0}} \text { for some } s \geq t\right\} \\
\leq & P_{y}\left\{\inf _{m<s \leq m+1}\left(R_{x}(s)-R_{x}(0)\right)\right. \\
& \left.\leq \frac{b_{0}(m+1)}{36 k_{0}} \text { for some } m>t-1\right\} \\
\leq & \sum_{m>t-1} P_{y}\left\{\frac{1}{3} b_{0} \mathscr{T}_{m}+\inf _{m<s \leq m+1}(\sqrt{2} w(s)) \leq \frac{b_{0}(m+1)}{36 k_{0}}\right\} \\
\leq & \sum_{m>t-1}\left(P_{y}\left\{\frac{1}{3} b_{0} \mathscr{T}_{m} \leq 2 \frac{\left.b_{0} m+1\right)}{36 k_{0}}\right\}\right. \\
& \quad P \sum_{m>t-1}\left(P_{y}\left\{\inf _{m}+\frac{(m+1)}{6 k_{0}}\right\}\right. \\
& \left.+P\left\{\sup _{m<s \leq m+1} w(s) \geq \frac{b_{0}(m+1)}{54 k_{0}}\right\}\right) \\
\leq & \sum_{m>t-1}\left(2^{2-k_{0}^{-1}(m-1)}+2 P\left\{w(m+1) \geq \frac{b_{0}(m+1)}{54 k_{0}}\right\}\right) \\
\leq & \left(2^{k_{0}^{-1}}-1\right)^{-1} 2^{2-k_{0}^{-1}(t-1)}+\sum_{m>t-1} \frac{108 k_{0}}{b_{0}} \exp \left(-\frac{b_{0}^{2}(m+1)}{2\left(54 k_{0}\right)^{2}}\right) \\
\leq & \frac{1}{2} \kappa^{-1} e^{-\kappa t} \quad
\end{aligned}
$$

for some $\kappa>0$, where we use that

$$
\int_{a}^{\infty} e^{-x^{2} / 2 s} d x \leq \int_{a}^{\infty} e^{-a x / 2 s} d x \leq \frac{2 s}{a} e^{-a^{2} / 2 s} .
$$

By Bishops's comparison theorem (see [Ch, pp. 71-73]),

$$
\sup \left\{Q_{x}(y): x, y \in M, \operatorname{dist}(x, y) \geq 1\right\}=b_{2}<\infty,
$$


where $b_{2}$ depends only on the lower bound of the Ricci curvature. Thus by (3.2)

$$
R_{x}(t)-R_{x}(0) \leq \sqrt{2} w(t)+b_{2} t+1, \quad t \leq \tau_{x}(1) .
$$

Again by the reflection principle (see [KS, p. 80]) and (3.16),

$$
\begin{aligned}
P_{y}\{ & \left.R_{x}(s)-R_{x}(0) \geq 2 b_{2} s \text { for some } s \geq t\right\} \\
& \leq P_{y}\left\{\sup _{m<s \leq m+1}\left(R_{x}(s)-R_{x}(0)\right) \geq 2 b_{2} m \text { for some } m>t-1\right\} \\
& \leq \sum_{m>t-1} P\left\{\sqrt{2} \sup _{m<s \leq m+1} w(s) \geq b_{2}(m-1)-1\right\} \\
& \leq \sum_{m>t-1} 2 P\left\{\sqrt{2} w(m+1) \geq\left(b_{2}(m-1)-1\right)\right\} \\
& \leq 4 \sum_{m>t-1}(m+1)^{1 / 2}\left(b_{2}(m-1)-1\right)^{-1} \exp \left(-\frac{\left(b_{2}(m-1)-1\right)^{2}}{4(m+1)}\right) \\
& \leq \frac{1}{2} \kappa^{-1} e^{-\kappa t}
\end{aligned}
$$

for an appropriately chosen $\kappa>0$ and $t>2 b_{2}^{-1}+2$. This, together with (3.16) yields (3.9) provided $\kappa$ is small enough.

3.4. Lemma. Let $\infty>r_{3}>r_{2}>r_{1}>0, x \in M, \xi \in S_{x} M$, and $y=$ $\operatorname{Exp}_{x}\left(r_{2} \xi\right)$. Then

$$
P_{y}\left\{\tau_{x}\left(r_{3}\right)>\tau_{x}\left(r_{1}\right)\right\} \leq P_{y}\left\{\infty>\tau_{x}\left(r_{1}\right)\right\} \leq 2 \kappa^{-1} \exp \left(-\frac{\kappa}{2}\left(r_{2}-r_{1}\right)\right)
$$

and for $t \geq \kappa^{-1}\left(r_{3}-r_{2}\right)$,

$$
P_{y}\left\{\tau_{x}\left(r_{3}\right) \geq t\right\} \leq \kappa^{-1} e^{-\kappa t},
$$

where $\kappa \leq \frac{1}{2}$ satisfies (3.9).

Proof. Note firstly that our choice of $\kappa \leq \frac{1}{2}$ means that $\kappa^{-1} e^{-\kappa}>1$ and hence that (3.9) holds for all $t$. By (3.2),

$$
R_{x}(t)-R_{x}(0) \geq \sqrt{2} w(t),
$$

and since $R_{x}(0)=r_{2}$ we derive by the submartingale inequality (see [Ks, p. 13]),

$$
\begin{aligned}
P_{y}\left\{\tau_{x}\left(r_{1}\right)\right. & \left.\leq \frac{1}{2}\left(r_{2}-r_{1}\right)\right\} \\
& =P_{y}\left\{\inf _{0 \leq s \leq\left(r_{2}-r_{1}\right) / 2}\left(R_{x}(s)-r_{2}\right) \leq r_{1}-r_{2}\right\} \\
& \leq P\left\{\inf _{0 \leq s \leq\left(r_{2}-r_{1}\right) / 2} w(s) \leq \frac{1}{\sqrt{2}}\left(r_{1}-r_{2}\right)\right\} \\
& =P\left\{\sup _{0 \leq s \leq\left(r_{2}-r_{1}\right) / 2} e^{w(s)} \geq \exp \left\{\frac{1}{\sqrt{2}}\left(r_{2}-r_{1}\right)\right)\right\} \\
& \leq e^{-\left(r_{2}-r_{1}\right) / 4} .
\end{aligned}
$$


On the other hand, by (3.9), $R_{x}(t)-R_{x}(0) \geq \frac{\kappa}{2}\left(r_{2}-r_{1}\right)$ for all $t \geq \frac{1}{2}\left(r_{2}-r_{1}\right)$, with probability at least $1-\kappa^{-1} \exp \left(-\frac{\kappa}{2}\left(r_{2}-r_{1}\right)\right)$, and so

$$
P_{y}\left\{\infty>\tau_{x}\left(r_{1}\right) \geq \frac{1}{2}\left(r_{2}-r_{1}\right)\right\} \leq \kappa^{-1} \exp \left(-\frac{\kappa}{2}\left(r_{2}-r_{1}\right)\right),
$$

which, together with (3.21), yields (3.19) provided $\kappa \leq \frac{1}{2}$. Next, by (3.9) for $\kappa t \geq r_{3}-r_{2}$,

$$
\begin{aligned}
P_{y}\left\{\tau_{x}\left(r_{3}\right) \geq t\right\} & \leq P_{y}\left\{R_{x}(t)-R_{x}(0)<r_{3}-r_{2}\right\} \\
& \leq P_{y}\left\{R_{x}(t)-R_{x}(0)<\kappa t\right\} \leq \kappa^{-1} e^{-\kappa t},
\end{aligned}
$$

proving (3.20).

The following lemma is similar to Lemma 2.3 of [KL]; it estimates the probability of the "angular" displacement of the Brownian motion.

3.5. Lemma. There exists $c>0$ such that if $x, \xi, y, r_{1}, r_{2}, r_{3}$ are as in Lemma 3.4, $r_{1} \geq r_{0} \geq 0$, and

$$
\begin{aligned}
& t_{x}\left(l ; r_{0}, r_{1}\right)=\inf \left\{\operatorname{dist}_{x}^{\left(r_{1}\right)}\left(\operatorname{Exp}_{x}\left(r_{1} \eta\right), \operatorname{Exp}_{x}\left(r_{1} \zeta\right)\right):\right. \\
& \left.\qquad, \zeta \in S_{x} M \text { and } \operatorname{dist}_{x}^{\left(r_{0}\right)}\left(\operatorname{Exp}_{x}\left(r_{0} \eta\right), \operatorname{Exp}_{x}\left(r_{0} \zeta\right)\right) \geq l\right\}^{1 / 2},
\end{aligned}
$$

then

$$
\begin{aligned}
& Q_{y}(x\left.; r_{0}, r_{1}, r_{3} ; l\right) \\
& \stackrel{\text { def }}{=} \\
& P_{y}\left\{\sup _{t: 0 \leq t \leq \tau_{x}\left(r_{1}\right) \wedge \tau_{x}\left(r_{3}\right)} \operatorname{dist}_{x}^{\left(r_{0}\right)}\left(\operatorname{Exp}_{x}\left(r_{0} \Xi_{x}(t)\right), \operatorname{Exp}_{x}\left(r_{0} \xi\right)\right) \geq l\right\} \\
& \leq P_{y}\left\{\tau_{x}\left(r_{1}\right) \wedge \tau_{x}\left(r_{3}\right)>t_{x}\left(l ; r_{0}, r_{1}\right)\right\} \\
&+c^{-1}\left(t_{x}\left(l ; r_{0}, r_{1}\right)\right)^{2} \exp \left(-c t_{x}\left(l ; r_{0}, r_{1}\right)\right)
\end{aligned}
$$

for any $l>0$ such that $t_{x}\left(l ; r_{0}, r_{1}\right) \geq 1$.

Proof. Put $t_{0}=t_{x}\left(l ; r_{0}, r_{1}\right)$. Then

$$
Q_{y}\left(x ; r_{0}, r_{1}, r_{3}, l\right) \leq P_{y}\left\{\tau_{x}\left(r_{1}\right) \wedge \tau_{x}\left(r_{3}\right)>t_{0}\right\}+\widetilde{Q}_{y},
$$

where

$$
\begin{aligned}
& \widetilde{Q}_{y}=P_{y}\left\{\underset{0 \leq t \leq \tau_{x}\left(r_{1}\right) \wedge \tau_{x}\left(r_{3}\right)}{\sup _{x}} \operatorname{dist}_{x}^{\left(r_{0}\right)}\left(\operatorname{Exp}_{x}\left(r_{0} \Xi_{x}(t)\right), \operatorname{Exp}_{x}\left(r_{0} \xi\right)\right) \geq l\right. \\
& \text { and } \left.\tau_{x}\left(r_{1}\right) \wedge \tau_{x}\left(r_{3}\right) \leq t_{0}\right\} \text {. }
\end{aligned}
$$

The event in the last probability can occur only if the Brownian motion $X(t)$ will cover distance at least $t_{0}^{2}$ in time not exceeding $t_{0}$, since by the Rauch comparison theorem any smooth curve lying entirely outside the ball $B_{x}\left(r_{1}\right)$ is at least as long as its projection to $S_{x}\left(r_{1}\right)$ along the geodesic rays through $x$. This means that $\tau_{y}\left(k_{0}\right) \leq t_{0}$ where $k_{0}=\left[t_{0}^{2}\right]$, and so

$$
\widetilde{Q}_{y} \leq P_{y}\left\{\tau_{y}\left(k_{0}\right) \leq t_{0}\right\}
$$

Define the random points $y_{i}, i=0, \ldots, k_{0}-1$, inductively by $y_{0}=y$, $y_{i+1}=X\left(\tau_{y_{i}}(1)\right)$. Then

$$
\tau_{y}\left(k_{0}\right) \geq \sum_{k_{0}-1 \geq i \geq 0} \tau_{y_{i}}(1)
$$


By Lemma 2.2 from [Ki] there exists a number $c_{1}>0$ such that for any $z \in M$ and $s>0$,

$$
P_{z}\left\{\tau_{z}(1) \leq s\right\} \leq c_{1}^{-1} \exp \left(-c_{1} s^{-1}\right) .
$$

Now by (3.26)-(3.28) and the strong Markov property,

$$
\begin{aligned}
\tilde{Q}_{y} & \leq P_{y}\left\{\sum_{l_{0}-1 \geq i \geq 0} \tau_{y_{i}}(1) \leq t_{0}\right\} \\
& \leq P_{y}\left\{\tau_{y_{i}}(1) \leq t_{0} k_{0}^{-1} \text { for some } i=0, \ldots, k_{0}-1\right\} \\
& \leq \sum_{l_{0}-1 \geq i \geq 0} E_{y} P_{y_{i}}\left\{\tau_{y_{i}}(1) \leq t_{0} k_{0}^{-1}\right\} \leq k_{0} \sup _{z \in M} P_{z}\left\{\tau_{z}(1) \leq t_{0} k_{0}^{-1}\right\} \\
& \leq k_{0} c_{1}^{-1} \exp \left(-c_{1} k_{0} t_{0}^{-1}\right) \leq c_{1}^{-1} t_{0}^{2} \exp \left(-c_{1}\left(t_{0}-1\right)\right)
\end{aligned}
$$

implying (3.24).

3.6. Lemma. Let $p(t, x, y)$ denote the heat kernel on $M$, i.e., the minimal positive fundamental solution of the parabolic equation $\partial u / \partial t=\Delta u$. There exists a number $a>0$ such that if $\operatorname{dist}(x, y)>1$ then

$$
p(t, x, y) \leq a^{-1} e^{-a \operatorname{dist}(x, y)} .
$$

Proof. The argument is the same as in [Ki, p. 204]. Recall that $p(t, x, y)$ is the transition density of the diffusion $X(t)$, and so by the strong Markov property

$$
\begin{aligned}
p(t, x, y) & =E_{x} \chi_{\tau_{y}(1)<t} p\left(t-\tau_{y}(1), X\left(\tau_{y}(1)\right), y\right) \\
& \leq C P_{x}\left\{\tau_{y}(1)<t\right\} \leq C P_{x}\left\{\tau_{y}(1)<\infty\right\}
\end{aligned}
$$

for some $C>0$ which by (3.19) gives (3.30).

\section{MAIN ReSUlts}

For $x \in M$ and $\xi \in S_{x} M$ let $\mathscr{C}_{x}(\xi, \varphi)$ denote the set of endpoints at $+\infty$ of the geodesics starting at $x$ whose initial velocity makes the angle less than $\varphi$ with $\xi$.

The Hausdorff dimension of a set $U \subset S(\infty)$ corresponding to a pole $x$ is given by

$$
\begin{aligned}
H d_{x}(U) & =\inf \left\{\delta \geq 0: \lim _{\rho \rightarrow 0} \inf \sum_{i} \varphi_{i}^{\delta}=0\right\} \\
& =\sup \left\{\delta \geq 0: \liminf _{\rho \rightarrow 0} \sum_{i} \varphi_{i}^{\delta}=\infty\right\},
\end{aligned}
$$

where the infimum inside the brackets is taken over all countable covers of $U$ by the sets

$$
V_{x}\left(\xi_{i}, \varphi_{i}\right)=\overline{\mathscr{C}_{x}\left(\xi_{i}, \varphi_{i}\right)} \cap S(\infty), \quad \xi_{i} \in S_{x} M, 0 \leq \varphi_{i} \leq \rho .
$$

The Hausdorff dimension of a probability measure $\mu$ on $S(\infty)$ is defined by

$$
H D_{x}(\mu)=\inf \left\{H d_{x}(U): U \subset S(\infty) \text { and } \mu(U)=1\right\} .
$$


Our main results correspond to the case where $M$ is the universal cover of a compact nonflat surface $N$ of nonpositive curvature which we will assume throughout this section. On the other hand what we really need for our method is that Lemmas 2.1 and 2.2 hold true and if one takes them as assumptions then the proof of Theorem 4.1 goes through without any further preconditions.

By [B], with probability one the limit

$$
\lim _{t \rightarrow \infty} \Xi_{x}(t)=\Xi_{x}(\infty)
$$

of the angular part of $X(t)$ exists, which in the two-dimensional case will follow also from the arguments of this section. The harmonic measures $P(x, \cdot)$ on $S(\infty)$ satisfy

$$
P(x, \Gamma)=P_{x}\left\{\Phi_{x} \Xi_{x}(\infty) \in \Gamma\right\}
$$

for any Borel $\Gamma \subset S(\infty)$ with the map $\Phi_{x}: S_{x} M \rightarrow S(\infty)$ defined in the introduction. In the same way as in [KL] the positivity of Hausdorff dimensions of $P(x, \cdot)$ will follow from the following result.

4.1. Theorem. There exists a number $\lambda>0$ and a sequence of numbers $C_{k}>0$ such that

$$
P_{x}\left\{\left\|\Xi_{x}(\infty, \omega)-\xi\right\| \leq \varphi \text { and } \omega \in \Omega_{x}^{(k)}\right\} \leq C_{k} \varphi^{\lambda}
$$

for any $x \in M, \xi \in S_{x} M$, and $\varphi \geq 0$ where $\Omega_{x}^{(k)}, k=1,2, \ldots$, is an increasing sequence of events such that $P_{x}\left(\bigcup_{k} \Omega_{x}^{(k)}\right)=1$.

4.2. Corollary. For any $x \in M$,

$$
H D_{x}(P(x, \cdot)) \geq \lambda \text {. }
$$

This corollary follows from Theorem 4.1 by a simple argument explained in $\S 3$ of [KL], and so we will need to prove only Theorem 4.1 itself. For any $x \in M, \zeta \in S_{x} M, t>0$, and $\rho>0$, define

$$
U_{t}(x, \xi, \rho)=\bigcup\left\{B_{z_{k}}(2 \rho): 0 \leq k \leq\left[t\left(2 \kappa^{-1}-\frac{1}{2} \kappa\right) \rho^{-1}\right]+1\right\}
$$

where $\kappa$ is the same as in (3.9), the balls $B_{z_{k}}(2 \rho)$ of radius $2 \rho$ are centered at the points $z_{k}=\operatorname{Exp}_{x}\left(\left(\frac{1}{2} \kappa t+k \rho\right) \xi\right)$, and [·] denotes the integral part. The main step in the proof of Theorem 4.1 is the following result (cf. Lemma 3.1 in $[\mathrm{KL}])$.

4.3. Proposition. For any $\delta>0$ there exists $q=q(\delta)>0$ such that

$$
P_{z}\left\{\sup _{t \geq 0} \operatorname{dist}_{x}^{(\rho)}\left(\operatorname{Exp}_{x}\left(\rho \Xi_{x}(t)\right), \operatorname{Exp}_{x}(\rho \xi)\right) \leq(\kappa \rho)^{\delta}\right\} \geq 1-q^{-1} \rho^{-q} e^{-q r}
$$

for all $x \in M, z=(\rho+r, \xi) \in S_{x}(\rho+r), \xi \in S_{x} M$, and $\rho \geq 1, r \geq 0$, where $\kappa$ is the same as in (3.9). Furthermore, for all $t>0$,

$$
\begin{aligned}
& P_{x}\left\{X(t) \in U_{t}\left(x, \Xi_{x}(\infty), t^{\delta}\right)\right\} \\
& \quad \begin{array}{l}
P_{x}\left\{\kappa t \leq R_{x}(t) \leq \kappa^{-1} t\right. \text { and } \\
\left.\quad \operatorname{dist}_{x}^{\left(R_{x}(t)\right)}\left(\operatorname{Exp}_{x}\left(R_{x}(t) \Xi_{x}(\infty)\right), X(t)\right) \leq t^{\delta}\right\} \geq 1-q^{-1} t^{-q} .
\end{array}
\end{aligned}
$$


Proof. We first derive (4.7) for $r=0$. It suffices to do this only for $\rho$ large enough and for $\delta \leq 1$ which we assume in what follows. For $n=1,2, \ldots$, put $l_{k}=\frac{1}{2}(\kappa \rho)^{(3 / 2) \delta}\left((\kappa \rho)^{\delta}+k^{2}\right)^{-1}$,

$$
W_{n}(z)=\left\{\eta \in S_{x} M: \operatorname{dist}_{x}^{(\rho)}\left(\operatorname{Exp}_{x}(\rho \xi), \operatorname{Exp}_{x}(\rho \eta)\right) \leq \sum_{k=1}^{n} l_{k}\right\}
$$

and

$$
D_{n}^{\varepsilon}(z)=\left\{\operatorname{Exp}_{x}(u \eta): \eta \in W_{n}(z), r_{\varepsilon}(n-1) \leq u \leq r_{\varepsilon}(n+1)\right\}
$$

where $r_{\varepsilon}(0)=\rho-\varepsilon \log \rho, r_{\varepsilon}(1)=\rho$, and $r_{\varepsilon}(n)=\rho+\varepsilon(n-1) \log \rho+\sum_{k=1}^{n-1} k^{2 / \delta}$ for $n>1$. We choose $\varepsilon$ so that $0<\varepsilon<1$ and for any $\eta_{1}, \eta_{2} \in S_{x} M$ and $u>0$,

$$
\begin{aligned}
& \operatorname{dist}_{x}^{(u+\varepsilon \log \rho)}\left(\operatorname{Exp}_{x}\left((u+\varepsilon \log \rho) \eta_{1}\right), \operatorname{Exp}_{x}\left((u+\varepsilon \log \rho) \eta_{2}\right)\right) \\
& \quad \leq(\kappa \rho)^{\delta / 4} \operatorname{dist}_{x}^{(u)}\left(\operatorname{Exp}_{x}\left(u \eta_{1}\right), \operatorname{Exp}_{x}\left(u \eta_{2}\right)\right) .
\end{aligned}
$$

For $n=1,2, \ldots$, set $\partial_{n}^{+}(z)=D_{n}^{\varepsilon}(z) \cap S_{x}\left(r_{\varepsilon}(n+1)\right), \partial_{n}^{-}(z)=D_{n}^{\varepsilon}(z) \cap$ $S_{x}\left(r_{\varepsilon}(n-1)\right)$, and $\partial_{n}^{\mathscr{E}}(z)=\partial D_{n}^{\varepsilon}(z) \backslash\left(\partial_{n}^{+}(z) \cup \partial_{n}^{-}(z)\right)$, where $\partial D$ denotes the boundary of $D$. The picture in $\S 3$ of [KL] makes the above notation more transparent.

Let $\tau_{n}=\inf \left\{t>0: X(t) \notin D_{n}^{\varepsilon}(z)\right\}$ be the exit time of the Brownian motion $X(t)$ from $D_{n}^{\varepsilon}(z)$. Then for any $y \in \partial_{n-1}^{+}$if $n>1$ and for $y=z$ if $n=1$ one has

$$
\begin{aligned}
P_{y}\left\{X\left(\tau_{n}\right) \notin \partial_{n}^{+}\right\} \leq & P_{y}\left\{\tau_{x}\left(r_{\varepsilon}(n+1)\right)>\tau_{x}\left(r_{\varepsilon}(n-1)\right)\right\} \\
& +P_{y}\left\{X\left(\tau_{n}\right) \in \partial_{n}^{\mathscr{E}}(x)\right\} .
\end{aligned}
$$

By (3.19),

$$
\begin{gathered}
\sup _{y \in S_{x}\left(r_{\varepsilon}(n)\right)} P_{y}\left\{\tau_{x}\left(r_{\varepsilon}(n+1)\right)>\tau_{x}\left(r_{\varepsilon}(n-1)\right)\right\} \\
\leq \kappa^{-1} \rho^{-\varepsilon \kappa / 2} \exp \left(-\frac{\kappa}{2}(n-1)^{2 / \delta}\right) .
\end{gathered}
$$

By (4.10) and the definition of $D_{1}^{\varepsilon}(z)$,

$$
P_{z}\left\{X\left(\tau_{1}\right) \in \partial_{1}^{\mathscr{E}}(z)\right\} \leq Q_{z}\left(x ; r_{\varepsilon}(0), r_{\varepsilon}(0), r_{\varepsilon}(2), \frac{1}{4}(\kappa \rho)^{\delta / 4}\right)
$$

with $Q_{y}$ defined by (3.24). Furthermore, by the definition of $D_{n}^{\varepsilon}(z), n=$ $2,3, \ldots$, for any $y \in \partial_{n-1}^{+}$,

$$
\begin{aligned}
& P_{y}\left\{X\left(\tau_{n}\right) \in \partial_{n}^{\mathscr{E}}(z)\right\} \\
& \quad \leq Q_{y}\left(x ; \rho, r_{\varepsilon}(n-1), r_{\varepsilon}(n+1), \frac{1}{2}(\kappa \rho)^{(3 / 2) \delta}\left((\kappa \rho)^{\delta}+n^{2}\right)^{-1}\right) .
\end{aligned}
$$

To employ (3.24) we have to estimate $t_{x}(l ; \rho, \rho+u)$ for certain $l>0$ and $u \geq$ 0 . Since the curvature is nonpositive then by the Rauch comparison theorem (see [Ch]), geodesics emanating from $x$ diverge at least as fast as in the zero curvature case, i.e., in the Euclidean plane. Thus for all $u>0$,

$$
\left(t_{x}(l ; \rho, \rho+u)\right)^{2} \geq(\rho+u) \rho^{-1} l .
$$

Put $u(l)=\max \left(0,2 R_{0} \rho l^{-1}-\rho\right)$. For $u=u(l)$, the right-hand side of $(4.15)$ is not less than $2 R_{0}$ and we can use Lemma 2.2 which yields that for any $u>u(l)$,

$$
\left(t_{x}(l ; \rho, \rho+u)\right)^{2} \geq 2 R_{0} \gamma_{0} \exp \left(\gamma_{0}(u-u(l))\right) .
$$


For $l_{n}$ defined before in (4.9) we have

$$
u\left(l_{n}\right)=\max \left(0,4 R_{0} \rho(\kappa \rho)^{-(3 / 2) \delta}\left((\kappa \rho)^{\delta}+n^{2}\right)-\rho\right)
$$

and so by (4.16) for $n \geq 3$ and all $\rho$ large enough

$$
\left(t_{x}\left(l_{n} ; \rho, r_{\varepsilon}(n-1)\right)\right)^{2} \geq 2 R_{0} \gamma_{0} \rho^{\varepsilon \gamma_{0}} \exp \left(\frac{\gamma_{0} \delta}{2(\delta+2)}(n-2)^{1+(2 / \delta)}\right) \text {. }
$$

Clearly, $t_{x}\left(l_{2} ; \rho, r_{\varepsilon}(1)\right)=l_{2}^{1 / 2}$ and $t_{x}\left(l_{1} ; r_{\varepsilon}(0), r_{\varepsilon}(0)\right)=l_{1}^{1 / 2}$.

Now (3.20), (3.24), (4.9)-(4.14), and (4.17) imply that (cf. (3.18) in [KL])

$$
P_{y}\left\{X\left(\tau_{n}\right) \notin \partial_{n}^{+}\right\} \leq c_{2}^{-1} \rho^{-c_{2}} e^{-c_{2} n}
$$

for some constant $c_{2}>0$ independent of $z, y \in M$ and $n \geq 1$ provided $y=z$ if $n=1$ and $y \in \partial_{n-1}^{+}$if $n>1$. Since $\sum_{n=1}^{\infty} l_{n} \leq(\kappa \rho)^{\delta},(4.18)$ together with the strong Markov property of $X(t)$ yields

$$
\begin{aligned}
& P_{z}\left\{\operatorname{dist}_{x}^{(\rho)}\left(\operatorname{Exp}_{x}\left(\rho \Xi_{x}(\infty)\right), z\right) \leq(\kappa \rho)^{\delta}\right\} \\
& \quad \geq P_{z}\left\{\sup _{t \geq 0} \operatorname{dist}_{x}^{(\rho)}\left(\operatorname{Exp}_{x}\left(\rho \Xi_{x}(t)\right), z\right) \leq(\kappa \rho)^{\delta}\right\} \\
& \quad \geq E_{z} \chi_{X\left(\tau_{1}\right) \in \partial_{1}^{+}} E_{X\left(\tau_{1}\right)} \chi_{X\left(\tau_{2}\right) \in \partial_{2}^{+}} \cdots \\
& \quad \geq 1-P_{z}\left\{X\left(\tau_{1}\right) \notin \partial_{1}^{+}\right\}-\sum_{n=2}^{\infty} \sup _{y \in \partial_{n-1}^{+}} P_{y}\left\{X\left(\tau_{n}\right) \notin \partial^{+}\right\} \\
& \quad \leq 1-c_{2}^{-2} \rho^{-c_{2}}
\end{aligned}
$$

implying (4.7) for $r=0$.

Now let $r>0$. Then using (4.19) with $\rho+r$ instead of $\rho$ we obtain by Lemma 2.2 that

$$
\begin{aligned}
P_{z}\left\{\sup _{t \geq 0} \operatorname{dist}_{x}^{(\rho)}\left(\operatorname{Exp}_{x}\left(\rho \Xi_{x}(t)\right), \operatorname{Exp}_{x}(\rho \xi)\right) \leq(\kappa \rho)^{\delta}\right\} \\
\quad \geq P_{z}\left\{\sup _{t \geq 0} \operatorname{dist}_{x}^{(\rho+r)}\left(\operatorname{Exp}_{x}\left((\rho+r) \Xi_{x}(t)\right), z\right) \leq(\kappa \rho)^{\delta} \gamma e^{\gamma r}\right\} \\
\geq 1-c_{2}^{-2} \gamma^{-c_{2}}(\kappa \rho)^{-c_{2} \delta} e^{-c_{2} \gamma r},
\end{aligned}
$$

provided $(\kappa \rho)^{\delta} \geq 2 R_{0}$ which we may assume to be true. Thus (4.7) follows for any $r \geq 0$. Finally, (3.9) and (4.19) together with the Markov property of $X(t)$ yield

$(4.21)$

$$
\begin{aligned}
& P_{x}\left\{X(t) \in U_{t}\left(x, \Xi_{x}(\infty), t^{\delta}\right)\right\} \\
& \quad \geq P_{x}\left\{\kappa t \leq R_{x}(t) \leq \kappa^{-1} t \text { and } \operatorname{dist}_{x}^{\left(R_{x}(t)\right)}\left(\operatorname{Exp}_{x}\left(R_{x}(t) \Xi_{x}(\infty), X(t)\right) \leq t^{\delta}\right\}\right. \\
& \geq \int_{B_{x}\left(\kappa^{-1} t\right) \backslash B_{x}(\kappa t)} P(t, x, d z) \\
& \quad \times P_{z}\left\{\operatorname{dist}_{x}^{\left(\rho_{x}(z)\right)}\left(\operatorname{Exp}_{x}\left(\rho_{x}(z) \Xi_{x}(\infty)\right), z\right) \leq\left(\kappa \rho_{x}(z)\right)^{\delta}\right\} \\
& \geq\left(1-c_{2}^{-2}(\kappa t)^{-c_{2}}\right)\left(1-\kappa^{-1} e^{-\kappa t}\right)
\end{aligned}
$$

implying (4.8) where $\rho_{x}(z)=\operatorname{dist}(x, z)$ and $P(t, x, \Gamma)=P_{x}\{X(t) \in \Gamma\}$.

Using (3.30) Theorem 4.1 follows from Proposition 4.3 proceeding verbatim as at the end of $\S 3$ in [KL]. 
Consider the measures $\mu_{x}=\Phi_{x} m_{x}$ introduced in $\S 1$. If $M$ is the universal cover of a compact surface $N$ of nonpositive curvature such that the geodesic flow on $N$ is ergodic then the stable and unstable foliations are absolutely continuous and we conclude that all the measures $\mu_{x}$ are equivalent. All the measures $P(x, \cdot)$ are equivalent by the Harnack inequality.

4.4. Theorem. If the geodesic flow on $N$ is ergodic then the measures $\mu_{x}$ and $P(x, \cdot)$ on $S(\infty)$ are singular unless the curvature is constant.

Proof. Let

$$
\beta=-\lim _{t \rightarrow \infty} \frac{1}{t} \log p(t, x, X(t))=\lim _{\delta \rightarrow 0} \liminf _{t \rightarrow \infty} \frac{1}{t} N(x, \delta, t),
$$

where $N(x, \delta, t)$ is the minimal cardinality of a finite set $E$ satisfying $P_{x}\{\operatorname{dist}(X(t), E) \leq 1\} \geq 1-\delta$. The main step in the proof of Theorem 4.4 which is similar to [L] is to show that if the measures $\mu_{x}$ and $P(x, \cdot)$ are equivalent, then $\beta=\alpha \gamma_{1}$, where $\alpha$ is from (3.4) and $\gamma_{1}$ is from Lemma 2.4. We will need the following proposition.

\subsection{Proposition. With probability 1}

$$
\lim _{t \rightarrow \infty} \frac{1}{t} \operatorname{dist}\left(X(t), \operatorname{Exp}_{x}\left(\alpha t \Xi_{x}(\infty)\right)\right)=0
$$

Proof. By (3.9), (4.7) and the Markov property of $X(t)$ we have

$$
\begin{array}{rl}
P_{x}\left\{\operatorname{dist}^{\left(R_{x}(t)\right)}\left(\operatorname{Exp}_{x}\left(R_{x}(t) \Xi_{x}(t+\varepsilon t)\right), \operatorname{Exp}_{x}\left(R_{x}(t) \Xi_{x}(\infty)\right)\right)>t^{\delta}\right\} \\
\leq & P_{x}\left\{\operatorname{dist}^{\left(R_{x}(t)\right)}\left(\operatorname{Exp}_{x}\left(R_{x}(t) \Xi_{x}(t+\varepsilon t)\right), \operatorname{Exp}_{x}\left(R_{x}(t) \Xi_{x}(\infty)\right)\right)\right. \\
\left.>\left(\kappa R_{x}(t)\right)^{\delta} \text { and } \kappa t \leq R_{x}(t) \leq \kappa^{-1} t\right\}+\kappa^{-1} e^{-\kappa t} & P(t, x, d y) \int_{M} P(\varepsilon t, y, d z) \\
= & \int_{B_{x}\left(\kappa^{-1} t\right) \backslash B_{x}(\kappa t)} P\left(\rho^{\prime}\right) \\
& \times P_{z}\left\{\operatorname{dist}^{\left(\rho_{x}(y)\right)}\left(\operatorname{Exp}_{x}\left(\rho_{x}(y) \xi_{x}(z)\right), \operatorname{Exp}_{x}\left(\rho_{x}(y) \Xi_{x}(\infty)\right)\right)\right. \\
\leq & \int_{B_{x}\left(\kappa^{-1} t\right) \backslash B_{x}(\kappa t)} P(t, x, d y) \int_{\left\{z: \kappa \varepsilon t \leq \rho_{x}(z)-\rho_{x}(y) \leq \kappa^{-1} \varepsilon t\right\}} P(\varepsilon t, y, d z) \\
& \times P_{z}\left\{\operatorname { s u p } _ { s \geq 0 } \operatorname { d i s t } ^ { ( \rho _ { x } ( y ) ) } \left(\operatorname{Exp}_{x}\left(\rho_{x}(y) \xi_{x}(z)\right),\right.\right. \\
& \left.\left.\quad \operatorname{Exp}_{x}\left(\rho_{x}(y) \Xi_{x}(s)\right)\right)>\left(\kappa \rho_{x}(y)\right)^{\delta}\right\} \\
& +\kappa^{-1} e^{-\kappa t}+\kappa^{-1} e^{-\kappa \varepsilon t} \leq q^{-1}(\kappa t)^{-q} e^{-q \kappa \varepsilon t}+\kappa^{-1} e^{-\kappa t}+\kappa^{-1} e^{-\kappa \varepsilon t},
\end{array}
$$

where $\xi_{x}(z) \in S_{x} M$ and $z=\operatorname{Exp}_{x}\left(\rho_{x}(z) \xi_{x}(z)\right)$. Thus by the Borel-Cantelli lemma for almost all $\omega$ there exists $N_{1}(\omega)$ such that for all $n \geq N_{1}(\omega)$,

$$
\operatorname{dist}^{\left(R_{x}(n, \omega)\right)}\left(\operatorname{Exp}_{x}\left(R_{x}(n, \omega) \Xi_{x}(n+\varepsilon n, \omega), \operatorname{Exp}_{x}\left(R_{x}(n, \omega) \Xi_{x}(\infty, \omega)\right)\right) \leq n^{\delta} .\right.
$$

It follows from (3.4) that for almost all $\omega$ there exists $N_{2}(\omega)$ such that for all $n \geq N_{2}(\omega)$,

$$
\begin{aligned}
& (1-\varepsilon) \alpha \leq n^{-1} R_{x}(n, \omega) \leq(1+\varepsilon) \alpha \quad \text { and } \\
& \alpha \leq n^{-1} R_{x}(n+\varepsilon n, \omega) \leq(1+2 \varepsilon) \alpha .
\end{aligned}
$$


Thus for all $n \geq N_{3}(\omega)=\max \left(N_{1}(\omega), N_{2}(\omega)\right)$,

$$
\operatorname{dist}\left(X(n+\varepsilon n, \omega), \operatorname{Exp}_{x}\left(\alpha n \Xi_{x}(\infty, \omega)\right)\right) \leq n^{\delta}+4 \varepsilon \alpha n .
$$

Furthermore, by (3.16), (3.18), the reflection principle, and the Markov property of $X(t)$,

$$
\begin{aligned}
P_{x}\left\{\sup _{0 \leq u \leq s} \operatorname{dist}(X(n), X(n+u))>l\right\} \\
\quad=\int_{M} P(n, x, d y) P_{y}\left\{\sup _{0 \leq u \leq s} \operatorname{dist}(y, X(u))>l\right\} \\
\quad \leq \sup _{y \in M} P_{y}\left\{\sup _{0 \leq u \leq s} R_{y}(u)>l\right\} \\
\quad \leq P\left\{\sup _{0 \leq u \leq s} w(u)>l-b_{2} s-1\right\}=2 P\left\{w(s)>l-b_{2} s-1\right\} \\
\quad \leq \frac{2 \sqrt{s}}{\left(l-b_{2} s-1\right)} \exp \left(-\frac{1}{2 s}\left(l-b_{2} s-1\right)\right) .
\end{aligned}
$$

We use (4.28) first with $s=\varepsilon n$ and $l=2 b_{2} \varepsilon n+1$ and then with $s=1$ and $l=$ $\varepsilon n$. For those values inequality (4.28) together with the Borel-Cantelli lemma yield that for almost all $\omega$ there exists $N_{4}(\omega)$ such that for all $n \geq N_{4}(\omega)$,

$$
\operatorname{dist}(X(n, \omega), X(n+\varepsilon n, \omega)) \leq 2 b_{2} \varepsilon n+1
$$

and

$$
\sup _{0 \leq u \leq 1} \operatorname{dist}(X(n), X(n+u)) \leq \varepsilon n .
$$

Since $\varepsilon>0$ arbitrary, by taking in (4.27) any positive $\delta<1$ we obtain (4.23) from (4.27), (4.29) and (4.30). Note that our proof, unlike [L], does not use directly the fact that $M$ is the universal cover of a compact manifold and it requires only (3.4) which may hold true in other circumstances, as well (see, for instance $[\mathrm{Pi}, \S \mathrm{V}])$.

The remaining part of the proof of Theorem 4.4 proceeds in the same way as in [L] employing the conformal equivalence of $M$ and the unit hyperbolic disk, the fact that conformal maps transform the Brownian motion into the time changed Brownian motion and certain inequalities connecting the numbers $\alpha, \beta$ and $\gamma_{1}$ before and after a conformal transformation. The proof of inequalities (a) and (b) on p. 192 in [L] remains the same in our case and inequality (c) based on Corollary 2.16 from [Kal] also holds true due to the ergodicity of the geodesic flow on $N$.

4.5. Remark. Note that for the universal cover of a compact negatively curved surface $S,[\mathrm{KL}]$ asserts that if the Hausdorff dimension of the harmonic measures is equal to one then the curvature is constant. This was proved by showing that the harmonic measures give rise to a certain measure invariant with respect to the geodesic flow on $S$ which is a Gibbs state corresponding to some Hölder continuous function and if the above Hausdorff dimension is one, then Pesin's formula holds true for this measure. By uniqueness, this measure must coincide with the Liouville measure. Therefore, the harmonic measure class is the 
same as geodesic measure class and by [L] the curvature is constant. In the nonpositive curvature case the uniqueness of an invariant measure satisfying Pesin's formula is not known, and we are not able to conclude that if the Hausdorff dimension is one for harmonic measures then the surface has constant curvature.

4.6. Remark. The method of the present paper works as well if one wants to describe the Martin boundary of $M$ with $\operatorname{dim} M=2$ in the same way as in [Ki]. It is necessary to adjust Lemmas $3.1,4.1$, and 4.2 from [Ki] in the spirit of the modifications we needed in $\S \S 3$ and 4 here as compared to the negative curvature case in [Ki and $\mathrm{KL}$ ]. In the two-dimensional case $M$ is conformally equivalent to the hyperbolic disk $D$ and hence the Laplace-Beltrami operator $\Delta_{M}$ on $M$ equals $\sigma \Delta_{D}$, where $\sigma$ is a positive function and $\Delta_{D}$ is the Laplace-Beltrami operator on $D$. Thus the harmonic functions, in particular the minimal positive harmonic functions, on $M$ and $D$ are essentially the same. However, the notion of the Martin boundary $\partial M$ involves not only minimal positive harmonic functions, but also the topology induced by Martin's functions $k_{y}(x)=g(x, y) / g\left(x_{0}, y\right)$, where $g(x, y)=\int_{0}^{\infty} p(t, x, y) d t$ is the Green's function. This topology is defined by saying that a sequence of points $y_{i} \in M$ is fundamental if the sequence $k_{y_{i}}$ converges to a harmonic function. Fundamental sequences are equivalent if their limiting functions coincide, and $\partial M$ is the set of equivalence classes. The topology on $M \cup \partial M$ is defined by the metric based on functions $k_{y}$ (see (4.7) in [Ki]). Since the Green's function $g$ on $M$ has no explicit connections with the Green's function on $D$, then, in general, the Martin topology on $M$ cannot be obtained via the conformal equivalence of $M$ and $D$. Thus it still makes sense to go all the way of [Ki] with the modifications as in the present paper even when $\operatorname{dim} M=2$ to derive that the Martin boundary $\partial M$ with the Martin topology is homeomorphic to a circle. The identification of the space of minimal positive harmonic functions on $M$ with a circle via the conformal equivalence of $M$ and $D$ corresponds, in general, to a different topology. Of course, the identification of the Martin boundary is more interesting in the full multidimensional generality of [B] where one cannot use the conformal equivalence, and so no description of the space of minimal positive harmonic functions is available at all.

\section{REFERENCES}

[B] W. Ballman, On the Dirichlet problem at infinity for manifolds of nonpositive curvature, Forum Math. 1 (1989), 201-203.

[BB] W. Ballman and M. Brin, On the ergodicity of geodesic flows, Ergodic Theory Dynamical Systems 2 (1982), 311-315.

[BBE] W. Ballman, M. Brin, and P. Eberlein, Structure of manifolds of nonpositive curvature. I, Ann. of Math. (2) 122 (1985), 171-203.

[CE] S. S. Chen and P. Eberlein, Isometry groups of simply connected manifolds of non-positive curvature, Illinois J. Math. 24 (1980), 73-103.

[Ch] I. Chavel, Eigenvalues in Riemannian geometry, Academic Press, Orlando, 1984.

[G] A. Gray, Tubes, Addison-Wesley, 1989.

[HI] E. Heintz and H. C. Im Hoff, Geometry of horospheres, J. Differential Geom. 12 (1977), 481-491.

[IW] N. Ikeda and S. Watanabe, Stochastic differential equations and diffusion processes, NorthHolland and Kodansha, 1981. 
[KS] I. Karatzas and S. E. Shreve, Brownian motion and stochastic calculus, Graduate Texts in Math., no. 113, Springer-Verlag, New York, 1988.

[Ka1] A. Katok, Entropy and closed geodesics, Ergodic Theory and Dynamical Systems 2 (1982), 339-367.

[Ka2] _ Four applications of conformal equivalence to geometry and dynamics, Ergodic Theory Dynamical Systems 8 (1988), 139-159.

[Ki] Y. Kifer, Brownian motion and positive harmonic functions on complete manifolds of nonpositive curvature, From Local Times to Global Geometry, Control and Physics (K. D. Elworthy, ed.), Pitman Research Notes in Math., no. 150, Longman, Harlow, 1986, pp. 187-232.

[KL] Y. Kifer and F. Ledrappier, Hausdorff dimension of harmonic measures on negatively curved manifolds, Trans. Amer. Math. Soc. 318 (1990), 685-704.

[L] F. Ledrappier, Propriété de Poisson et courbure négative, C. R. Acad. Sci. Paris Ser. I 305 (1987), 191-194.

[P] Ja. B. Pesin, Geodesic flows on closed Riemannian manifolds without focal points, Math. USSR Izv. 11 (1977), 1195-1228.

[Pi] M. A. Pinsky, Stochastic Riemannian geometry, Probabilistic Analysis and Related Topics, vol. 1 (A. T. Bharucha-Reid, ed.), Academic Press, New York, 1978, pp. 199-236.

[Va] N. T. Varopoulos, Information theory and harmonic functions, Bull. Sci. Math. (2) 110 (1986), 347-389.

Department of Mathematics, University of Maryland, College Park, Maryland 20742

E-mail address: mbrin@math.umd.edu

Institute of Mathematics, Hebrew University of Jerusalem, Jerusalem, IsRael

E-mail address: kifer@math.huji.ac.il 